\title{
On Stability Analyses of Three Classical Buckling Problems for the Elastic Strut
}

\author{
Oliver M. O'Reilly • Daniel M. Peters
}

Received: 16 October 2010 / Published online: 13 January 2011

(C) The Author(s) 2011. This article is published with open access at Springerlink.com

\begin{abstract}
It is common practice in analyses of the configurations of an elastica to use Jacobi's necessary condition to establish conditions for stability. Analyses of this type date to Born's seminal work on the elastica in 1906 and continue to the present day. Legendre developed a treatment of the second variation which predates Jacobi's. The purpose of this paper is to explore Legendre's treatment with the aid of three classical buckling problems for elastic struts. Central to this treatment is the issue of existence of solutions to a Riccati differential equation. We present two different variational formulations for the buckling problems, both of which lead to the same Riccati equation, and we demonstrate that the conclusions from Legendre and Jacobi's treatments are equivalent for some sets of boundary conditions. In addition, the failure of both treatments to classify stable configurations of a free-free strut are contrasted.
\end{abstract}

Keywords Elastica $\cdot$ Variational Methods $\cdot$ Elastic Stability $\cdot$ Buckling

Mathematics Subject Classification (2000) 34B15 - 49B10 · 74G60 - 74K10 • 93C10

\section{Introduction}

Euler's theory of the elastica has been applied since the mid-1740s to determine planar configurations of long, slender, flexible rods which are subject to terminal loading (see [14] and references therein). It is arguably the simplest nonlinear rod theory and has been used to provide elegant demonstrations of buckling phenomena and adhesion problems. Of particular interest in this paper is the manner in which nonlinear stability of the straight and buckled configurations of three classical elastic strut problems are determined. The three problems are discussed in Love [14, Sect. 264] and illustrated in Fig. 1. The problems feature uniform rods of constant flexural rigidity $E I$ and length $\ell$ which are subject to compressive

O.M. O’Reilly $(\varangle) \cdot$ D.M. Peters

Department of Mechanical Engineering, University of California at Berkeley, Berkeley, CA 94720-1740, USA

e-mail: oreilly@berkeley.edu 
(a)

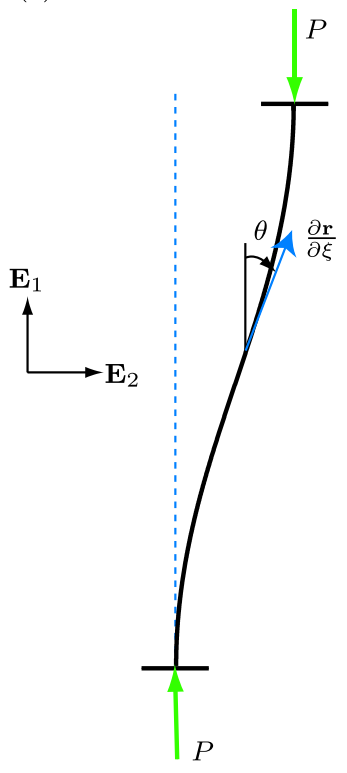

(b)

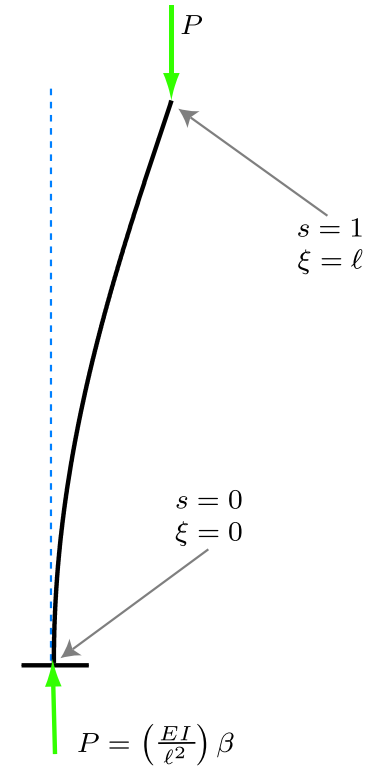

(c)

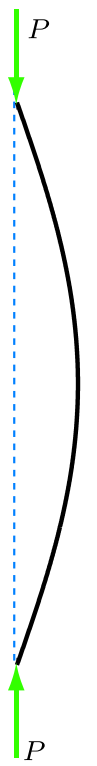

Fig. 1 Schematic of an elastic rod under dimensionless axial load $\beta$ for three sets of boundary conditions: (a) fixed-fixed (also known as Dirichlet-Dirichlet), (b) fixed-free (also known as Dirichlet-Neumann), and (c) free-free (also known as Neumann-Neumann)

terminal forces $P$. Depending on the boundary conditions, as $P$ is increased beyond a critical load value $P_{\text {crit }}$, the straight rod buckles and two stable configurations are created in the bifurcation.

It is easy to determine the buckled states of the strut. Using the kinetic analogue with the planar pendulum, the boundary value problem readily lends itself to analysis and predicting the trivial and buckled states is straightforward. Establishing the nonlinear stability of the straight and buckled configurations is non-trivial. One avenue is to determine which configurations minimize the total energy of the rod. Dating to Born's seminal thesis [3] in 1906 and continuing to this day (cf. $[8,12,15,16,23]$ ), the main avenue to quantify whether or not the total energy is minimized is to use Jacobi's necessary condition. ${ }^{1}$ That is, one examines an accessory variational problem and determines conditions for the nonexistence of conjugate points.

For the fixed-fixed and fixed-free cases shown in Fig. 1(a, b), the search for conjugate points produces results which are in agreement with the classical predictions of the critical load $P_{\text {crit }}$. However, as noted recently by Manning [16], a similar analysis for the free-free case will lead to erroneous conclusions. ${ }^{2}$ Surprised by this result, we were lead to examine an earlier necessary condition for the positive definiteness of the second variation that was developed by Legendre in 1786. His treatment results in a Riccati differential equation and the search for bounded solutions to it. One of the goals of this paper is to examine the appli-

\footnotetext{
${ }^{1}$ There have also been numerous reinterpretations of, and extensions to, Jacobi's condition since he initially proposed it in 1837 (see [16, 17, 21, 24] and references therein).

${ }^{2}$ An alternative treatment of the second variation for the free-free case can be found in recent works by Kuznetsov and Levyakov [10, 13].
} 
cation of Legendre's treatment to buckling problems. It will come as no surprise to readers familiar with the excellent expositions of Legendre's work in Bolza [2] and Gelfand and Fomin [6] that the results from Legendre's treatment are equivalent to the Jacobi treatment for the fixed-fixed elastic strut. We also demonstrate their equivalence for the fixed-free strut. For the free-free elastic strut, it is easy to see that a solution of the Riccati equation associated with Legendre's treatment does not exist, and thus it is impossible to make the erroneous conclusions associated with a naive treatment based solely on conjugate points.

Legendre's treatment of the second variation has attracted considerable attention in the field of optimal control (see [4] for example). We thus present two variational formulations of the buckling problem. The first of these, which is presented in Sect. 3, is a classical treatment, while the second formulates the problem as an optimal control problem. However, in contrast to the control-based formulations of Bryson and Ho [4, p. 191] and Sachkov $[22,23]$, the resulting formulation results in a singular control problem. With the significant assistance of works by Bell and Jacobson [1], we succeed in Sect. 4 in showing that the necessary condition for optimality reduces to the same Riccati equation found with the earlier formulation.

In Sect. 5, the Riccati equation ((17) or $\left.(59)_{1}\right)$ is examined for all three classical buckling problems, and sufficient conditions for the stability of the straight and buckled configurations to the strut are established. Furthermore, the absence of a solution to the Riccati equation for the free-free strut is a clear indication of the degenerate nature of this case and the need for a modified treatment of the type proposed recently by Manning [16] (see also Kuznetsov and Levyakov [10,13]). We close the paper with a discussion of future applications to Legendre's treatment to the stability of branched equilibria of elastic rods such as those discussed in $[5,19]$.

\section{The Elastic Strut and the Three Buckling Problems}

The problems of interest feature the deformed planar shape of a long, slender, uniform rod which has a flexural rigidity of $E I$ and a fixed length $\ell$ (cf. Fig. 1). The ends of the rod are defined by the material coordinates $\xi=0$ and $\xi=\ell$, and, in its fixed reference configuration, the centerline of the rod is parallel to the constant unit vector $\mathbf{E}_{1}: \mathbf{R}=\xi \mathbf{E}_{1}$, where $\mathbf{R}$ is the position vector of a point on the centerline. A constant force $P \mathbf{E}_{1}$ is applied at $\xi=0$ and a force $-P \mathbf{E}_{1}$ is applied at $\xi=\ell$. Here, $\left\{\mathbf{E}_{1}, \mathbf{E}_{2}, \mathbf{E}_{3}=\mathbf{E}_{1} \times \mathbf{E}_{2}\right\}$ denotes a fixed Cartesian basis for $\mathbb{E}^{3}$.

The deformed configuration of the rod is defined by the vector-valued function $\mathbf{r}(\xi)=$ $X \mathbf{E}_{1}+Y \mathbf{E}_{2}$. The unit tangent vector to the deformed centerline has the representations

$$
\frac{\partial \mathbf{r}}{\partial \xi}=\frac{\partial X}{\partial \xi} \mathbf{E}_{1}+\frac{\partial Y}{\partial \xi} \mathbf{E}_{2}=\cos (\theta) \mathbf{E}_{1}+\sin (\theta) \mathbf{E}_{2} .
$$

The bending moment $\mathbf{m}=M \mathbf{E}_{3}$ in the rod is proportional to the curvature $\frac{\partial \theta}{\partial \xi}$ :

$$
M=E I \frac{\partial \theta}{\partial \xi} .
$$

For a terminally loaded rod, the equations governing the elastica reduce to

$$
\mathbf{n}=-P \mathbf{E}_{1}, \quad \frac{\partial M}{\partial \xi}+\left(\left(\cos (\theta) \mathbf{E}_{1}+\sin (\theta) \mathbf{E}_{2}\right) \times \mathbf{n}\right) \cdot \mathbf{E}_{3}=0,
$$


where $\mathbf{n}$ is the contact force. This pair of equations can be reduced to a second-order ordinary differential equation for $\theta(\xi)$ :

$$
E I \frac{\partial^{2} \theta}{\partial \xi^{2}}+P \sin (\theta)=0
$$

Thus, they need to be supplemented by boundary conditions on $\theta$ and $\frac{\partial \theta}{\partial \xi}$. Analytical expressions for $\theta(s), X(s)$, and $Y(s)$ for all the solutions to (4) that are discussed in this paper can be found in Love [14, Sect. 263].

By summing the potential energies of the terminal loads and the strain energy of the rod, the following expression for the total energy of the rod can be computed:

$$
E=\int_{\xi=0}^{\xi=\ell} \frac{E I}{2}\left(\frac{\partial \theta}{\partial \xi}\right)^{2}+P \cos (\theta) d \xi .
$$

It is well known that (4) are the Euler-Lagrange differential equations associated with seeking extremizers of $E$.

It is convenient to non-dimensionalize the governing equations (4) with the help of the coordinate $s$ and parameter $\beta$ :

$$
s=\frac{\xi}{\ell}, \quad \beta=\frac{P \ell^{2}}{E I} .
$$

The ends of the rod can now be denoted by $s=s_{0}$ and $s=s_{f}$ where $s_{0}=0$ and $s_{f}=1$. Further, (4) simplifies to

$$
\theta^{\prime \prime}+\beta \sin (\theta)=0,
$$

where the prime denotes the partial derivative with respect to $s$.

Referring to Fig. 1, we are interested in three sets of boundary conditions in the sequel. In the first case, the ends of the rod are clamped. This case is called the fixed-fixed case or the Dirichlet-Dirichlet (DD) case. For the second case, which is called the fixed-free case or the Dirichlet-Neumann (DN) case, the end $s=0$ is clamped while the end at $s=1$ is free of applied moments. Finally, in the third case, which we refer to as the free-free case or the Neumann-Neumann (NN) case, both ends of the rod are free of applied moments. In summary,

$$
\begin{array}{ll}
\text { DD: } & \theta(s=0)=0, \quad \text { and } \quad \theta(s=1)=0, \\
\text { DN : } & \theta(s=0)=0, \quad \text { and } \quad \theta^{\prime}(s=1)=0, \\
\text { NN : } & \theta^{\prime}(s=0)=0, \quad \text { and } \quad \theta^{\prime}(s=1)=0 .
\end{array}
$$

In the sequel, we consider the solution to (7) subject to boundary conditions of the form

$$
\phi\left[\theta\left(s_{0}\right), \theta^{\prime}\left(s_{0}\right)\right]=0, \quad \psi\left[\theta\left(s_{f}\right), \theta^{\prime}\left(s_{f}\right)\right]=0,
$$

where $\phi$ and $\psi$ are smooth scalar-valued functions. Clearly (9) encompass the three sets of conditions listed in (8).

\section{A Classical Variational Formulation}

One popular subject in elastic stability is to consider the variational problem of extremizing the integral of the energy subject to boundary conditions of the form (9). Formulations of 
this type are classical and the development of necessary conditions for the minimization of the energy continue to this date. Here, we discuss the necessary conditions and place particular emphasis on Legendre and Jacobi's examination of the second variation of the energy. The application of Legendre's formulation is one of particular interest and will be applied in Sect. 5 .

We denote the dimensionless energy functional obtained from (5) by $I$ :

$$
I=\int_{0}^{1}\left(\frac{1}{2}\left(\theta^{\prime}\right)^{2}+\beta \cos (\theta)\right) d s .
$$

It is easy to see that the Euler-Lagrange equations associated with setting the first variation $\delta I=0$ are equivalent to (7). We denote the solution to (7) with an asterix: $\theta=\theta^{*}(s)$. The second variation of $I$ about an extremum $\theta=\theta^{*}(s)$ can be computed in a standard manner:

$$
\delta^{2} I=2 \epsilon^{2} \int_{0}^{1} \Omega(\eta) d s
$$

where

$$
2 \Omega=-\beta \cos \left(\theta^{*}\right) \eta^{2}+\eta^{\prime 2},
$$

and $\eta$ is a variation of $\theta, \theta=\theta^{*}+\epsilon \eta(s)$, which satisfies the appropriate boundary conditions (from (9)):

$$
\frac{\partial \phi}{\partial \theta} \eta(0)+\left.\frac{\partial \phi}{\partial \theta^{\prime}} \eta^{\prime}(0)\right|_{\theta=\theta^{*}(0)}=0, \quad \frac{\partial \psi}{\partial \theta} \eta(1)+\left.\frac{\partial \psi}{\partial \theta^{\prime}} \eta^{\prime}(1)\right|_{\theta=\theta^{*}(1)}=0 .
$$

\subsection{The Necessary Condition L1}

A necessary condition for $\theta^{*}(s)$ to minimize $I$ is that

$$
\delta^{2} I \geq 0,
$$

for all sufficiently smooth $\eta(s)$ which satisfy (13). To check the positivity of the second variation, one approach, dating to Legendre in 1786 [2], is to add the following identity to (11):

$$
\int_{0}^{1}\left(\eta^{2} w\right)^{\prime} d s-\left[\eta^{2} w\right]_{s=0}^{s=1}=0
$$

Thus, $\delta^{2} I$ simplifies to

$$
\delta^{2} I=\frac{\epsilon^{2}}{2} \int_{0}^{1}\left(\eta^{\prime}+w \eta\right)^{2} d s-\epsilon^{2}\left[\eta^{2} w\right]_{s=0}^{s=1}
$$

provided we can find a function $w(s)$ which satisfies the following Riccati equation

$$
w^{\prime}=w^{2}+\beta \cos \left(\theta^{*}\right) .
$$

Hence, assuming we can find a solution $w(s)$ of the Riccati equation where the boundary condition on $w(s)$ is chosen so that

$$
\left[\eta^{2} w\right]_{s=0}^{s=1} \leq 0,
$$

we can conclude that $\theta^{*}(s)$ satisfies a necessary condition to minimize the total energy of the rod. We denote this condition as L1. 


\subsection{The Necessary Condition J1}

Building on Legendre's earlier work, an alternative treatment of $\delta^{2} I$ was proposed by Jacobi in 1837 [2]. For his treatment, one manipulates (11) and expresses it in the form

$$
\delta^{2} I=\frac{\epsilon^{2}}{2} \int_{0}^{1} \eta\left(-\eta^{\prime \prime}-\beta \cos \left(\theta^{*}\right) \eta\right) d s+\epsilon^{2}\left[\eta \eta^{\prime}\right]_{s=0}^{s=1} .
$$

Notice that the second term on the right-hand side will vanish for each of the three sets of boundary conditions (8). Jacobi's necessary condition for solutions $\theta^{*}$ to minimize $I$ is the non-existence of conjugate points to $s=0$. For the elastica, this criterion reduces to examining solutions $\eta(s)$ to Jacobi's equation,

$$
\eta^{\prime \prime}+\beta \cos \left(\theta^{*}\right) \eta=0
$$

which satisfy the boundary conditions $(8)_{1,2}$. We refer to this necessary condition for the minimization of $I$ as J1. Jacobi also showed that (17) and (20) are related by the transformation $\eta^{\prime}=-w \eta$. A good discussion on the relationship between $\mathrm{J} 1$ and $\mathrm{L} 1$ can be found in [6, Chap. 5].

For fixed-fixed and fixed-free struts, the estimates found using $\mathrm{J} 1$ agree with classical buckling results for the strut (see, e.g., [3, 15]). However, for the free-free strut (NN), a naive application of the Jacobi condition yields estimates which do not agree with classical buckling results. Manning [16] recently presented a modified version of J1 which accommodates the free-free boundary conditions by explicitly eliminating solutions of the type (21). However, it is not obvious a priori why J1, which works so successfully for the fixed-free and fixed-fixed cases, should fail for the free-free case.

The easiest method to see why $\mathrm{J} 1$ fails for the free-free strut which is straight (i.e., $\theta^{*}=0$ ) is to consider the following variation to $\theta^{*}=0:^{3}$

$$
\eta(s)=a,
$$

where $a$ is a constant. Clearly, (21) satisfies the boundary conditions $\eta^{\prime}(0)=0$ and $\eta^{\prime}(1)=$ $0 .{ }^{4}$ Moreover, after substituting (21) into either (11) or (19) we find that

$$
\delta^{2} I=-\frac{\epsilon^{2} \beta a^{2}}{2}<0
$$

Hence, $\theta^{*}=0$ does not minimize $I$ when $\beta \geq 0$. However, this conclusion is at odds with classical buckling results which state that the straight configuration is stable provided $\beta<\pi^{2}$. We explore this problem in further detail in Sect. 5.3. There it will become apparent how $\mathrm{L} 1$ readily indicates that there is an issue with the free-free strut.

\footnotetext{
${ }^{3}$ The solution (21) is known as the negative inborn eigenfunction of the Jacobi equation in [16]. In the treatment of the second variation by Kuznetsov and Levyakov [10, 13], variations of this type are excluded by imposing the isoperimetric constraint $\int_{0}^{1} \cos (\theta) d s=1$.

${ }^{4}$ The function $\eta(s)=a$ does not satisfy the Jacobi equation (20). Further, the configuration $\theta(s)=\epsilon a \neq 0$ does not satisfy the equilibrium conditions (4). Thus, neither searching for conjugate points nor evaluating $I$ for particular equilibrium configurations will help to illuminate the degenerate nature of the free-free strut.
} 


\subsection{Other Necessary and Sufficient Conditions}

One of the three remaining necessary conditions for an extremum is due to Weierstrass. For the elastic struts of interest in this paper, it is easy to compute the Weierstrass excess function:

$$
\mathcal{E}\left(s, \theta, \theta^{\prime}, p\right)=\frac{1}{2}\left(\theta^{\prime}-p\right)^{2},
$$

where $p$ is a real number. Thus, $\mathcal{E}$ is positive definite and so we conclude that the Weierstrass necessary condition is trivially satisfied. The remaining pair of necessary conditions are the Erdmann-Weierstrass corner conditions. For the elastica strut these reduce to showing that extremizers of $I$ have continuous slopes $\theta^{\prime}$, and are identically satisfied by the solutions of interest in this paper.

Assuming that the Euler-Lagrange differential equation is satisfied by $\theta^{*}(s)$, and given that the Weierstrass and Erdmann-Weierstrass conditions are satisfied, it is well-known that satisfaction of either $\mathrm{J} 1$ (where applicable) ${ }^{5}$ or L1 is sufficient for $\theta^{*}(s)$ to be a weak minimum of $I$ (see, e.g., $[2,6]$ ).

\section{Optimal Control Formulation}

An alternative variational formulation for the equilibrium configurations of the elastic strut can be found when the problem of determining these configurations is formulated as an optimal control problem. In contrast to the related control-based formulation of Bryson and Ho [4, p. 191] and Sachkov [22, 23], the resulting formulation results in a singular control problem. However, with the significant assistance of works by Bell and Jacobson [1], we are able to show the equivalence of this formulation to the one presented in Sect. 3. Furthermore, one of the necessary conditions for optimality is identical to the Riccati equation (17) associated with the condition L1 discussed in Sect. 3.1.

Proceeding with the formulation, we seek to compute the control $u$ which extremizes the cost functional

$$
J=F\left[\mathbf{x}\left(s_{0}\right)\right]+G\left[\mathbf{x}\left(s_{f}\right)\right]+\int_{s_{0}}^{s_{f}} L(\mathbf{x}, u, s) d s
$$

subject to the following conditions:

$$
\mathrm{x}^{\prime}=f(\mathrm{x}, u, s), \quad \phi\left[\mathrm{x}\left(s_{0}\right)\right]=0, \quad \psi\left[\mathrm{x}\left(s_{f}\right)\right]=0 .
$$

The state vector $\mathrm{x}$ is $n$-dimensional, $u$ is a scalar control variable, $\phi$ and $\psi$ are smooth scalar functions of the initial and final states $\times\left(s_{0}\right)$ and $\times\left(s_{f}\right)$, respectively, and $F$ and $G$ are scalarvalued functions representing the initial and final costs. The initial constraint $\phi$ is not typical in optimal control formulations but is necessary in the problems of interest here because the components of $\mathrm{x}\left(s_{0}\right)$ are generally not all specified.

We can adjoin equations (25) to the cost functional with Lagrange multipliers $\lambda(s), v_{0}$, and $v_{f}$. This yields the augmented cost functional $\bar{J}$ :

$$
\bar{J}=F\left[\mathrm{x}\left(s_{0}\right)\right]+v_{0} \phi\left[\mathrm{x}\left(s_{0}\right)\right]+G\left[\mathrm{x}\left(s_{f}\right)\right]+v_{f} \psi\left[\mathrm{x}\left(s_{f}\right)\right]
$$

\footnotetext{
${ }^{5}$ Recall that it is known that the search for conjugate points of Jacobi's equation (20) is not applicable to the free-free rod [16].
} 


$$
+\int_{s_{0}}^{s_{f}}\left[H(\mathrm{x}, u, \lambda, s)-\lambda(s) \cdot \mathrm{x}^{\prime}\right] d s,
$$

where the Hamiltonian

$$
H=L+\lambda \cdot f
$$

In the elastic strut problem, the two state variables are $\theta$ and $\theta^{\prime}$ :

$$
x_{1}=\theta, \quad x_{2}=\theta^{\prime} .
$$

The dimensionless arc-length $s$ becomes the independent variable such that $s_{0}=0$ and $s_{f}=1$. Therefore, the problem is to minimize

$$
J=\int_{0}^{1}\left(\frac{1}{2} x_{2}^{2}+\beta \cos \left(x_{1}\right)\right) d s,
$$

subject to

$$
x_{1}^{\prime}=x_{2}, \quad x_{2}^{\prime}=u,
$$

and appropriate boundary conditions (9) on $\times(s)$. Note that the terminal costs $F$ and $G$ are generally identically zero for the elastica problems of interest here. The Hamiltonian for the problem is defined as

$$
H(\mathrm{x}, u, \lambda, s)=\frac{1}{2} x_{2}^{2}+\beta \cos \left(x_{1}\right)+\lambda_{1} x_{2}+\lambda_{2} u .
$$

Comparing (7) to (30), it is important to note that we have chosen to specify the vector field $\mathrm{f}$ as $\mathrm{f}=\left[\begin{array}{ll}x_{2} & u\end{array}\right]^{T}$ rather than $\mathrm{f}=\left[\begin{array}{ll}x_{2}-\beta \sin \left(x_{1}\right)\end{array}\right]^{T}$. It will shortly become apparent that the control variable $u$ corresponds to the (dimensionless) moment of the applied force $P$.

\subsection{First Variation}

We now follow a standard procedure (see, e.g., [4]) and consider the first variation $\delta \bar{J}$ of $\bar{J}$ with changes in the control $u$ for fixed endpoints $s_{0}$ and $s_{f}$. The change in $u$ causes changes in $x$ and consequently

$$
\begin{aligned}
\delta \bar{J}= & {\left[\left(F_{\mathrm{x}}+v_{0} \phi_{\mathrm{x}}+\lambda\right) \delta \mathbf{x}\right]_{s=s_{0}}+\left[\left(G_{\mathrm{x}}+v_{f} \psi_{\mathrm{x}}-\lambda\right) \delta \mathbf{x}\right]_{s=s_{f}} } \\
& +\int_{s_{0}}^{s_{f}}\left[\left(H_{\mathrm{x}}+\lambda^{\prime}\right) \delta \mathbf{x}+H_{u} \delta u\right] d s .
\end{aligned}
$$

The subscript $\mathrm{x}$ and $u$ on a function denote partial derivatives of the function with respect to these variables. By requiring that $\delta \bar{J}$ vanish for arbitrary $\delta u$, we obtain necessary conditions for optimality:

$$
\begin{aligned}
& \lambda^{\prime}=-H_{\mathrm{x}}, \quad H_{u}=0, \\
& \lambda\left(s_{0}\right)=-\left(F_{\mathrm{x}}+v_{0} \phi_{\mathrm{x}}\right)_{s=s_{0}}, \quad \lambda\left(s_{f}\right)=\left(G_{\mathrm{x}}+v_{f} \psi_{\mathrm{x}}\right)_{s=s_{f}} .
\end{aligned}
$$

These equations are supplemented by the state equations and boundary conditions (25):

$$
\mathrm{x}^{\prime}=H_{\lambda}, \quad \phi\left[\mathrm{x}\left(s_{0}\right)\right]=0, \quad \psi\left[\mathrm{x}\left(s_{f}\right)\right]=0 .
$$


The identity $H_{u}=0$ generally serves to define the optimal control $u^{*}$, while the differential equations $\mathrm{x}^{\prime}=H_{\lambda}$ and $\lambda^{\prime}=-H_{\mathrm{x}}$ are used to compute the optimal solutions $\lambda^{*}(s)$ and $\mathrm{x}^{*}(s)$. In the sequel, we avoid ornamenting the optimal solution with an asterix unless it is necessary to avoid confusion. The boundary conditions on $\lambda(s)$ and the boundary conditions on $\mathbf{x}(s)$ (which are presented in $(33)_{3,4}$ and $(34)_{2,3}$ ) total $2 n$ in number and are intended to make the differential equations $\lambda^{\prime}=-H_{\mathrm{x}}$ and $\mathrm{x}^{\prime}=H_{\lambda}$ well-posed. ${ }^{6}$

It is important to note that the Hamiltonian defined in (31) is linear in the control $u$. This implies that the optimal control problem is singular, meaning that the standard optimal control formulation where Pontryagin's Minimum Principle is invoked to determine the optimal control $u^{*}$ is of no assistance in finding the optimal control $u^{*}$. For this reason, starting in the 1960s new necessary conditions for singular optimal control problems were established by a number of researchers (see $[1,4,7,18]$ and references therein).

The first of the aforementioned necessary conditions is known as the generalized Legendre-Clebsch condition and is defined as

$$
(-1)^{q} \frac{\partial}{\partial u}\left[\left(\frac{d^{2 q}}{d s^{2 q}}\right) H_{u}\right] \geq 0,
$$

where $2 q$ is the lowest-order derivative of $H_{u}$ in which $u$ appears explicitly. The second necessary condition is known as the Jacobson condition [7], and it requires that

$$
\begin{gathered}
\mathrm{f}_{u} \cdot\left[F_{\mathrm{xx}}+\left(v_{0} \phi_{\mathrm{x}}\right)_{\mathrm{x}}\right]+\left.H_{u \mathrm{x}}\right|_{\left(\mathrm{x}=\mathrm{x}^{*}\left(s_{0}\right), u=u^{*}\left(s_{0}\right)\right)}=0, \\
\mathrm{f}_{u} \cdot\left[G_{\mathrm{xx}}+\left(v_{f} \psi_{\mathrm{x}}\right)_{\mathrm{x}}\right]+\left.H_{u \mathrm{x}}\right|_{\left(\mathrm{x}=\mathrm{x}^{*}\left(s_{f}\right), u=u^{*}\left(s_{f}\right)\right)}=0 .
\end{gathered}
$$

Finally, to determine the optimal control in singular problems, a series of partial derivatives of $H$ are computed until $u^{*}$ can be prescribed: ${ }^{7}$

$$
H_{u}=0, \quad H_{u}^{\prime}=0, \quad H_{u}^{\prime \prime}=0, \ldots
$$

\subsubsection{Application to the Elastica}

Applying the necessary conditions (33) to the elastic strut problem, we obtain the state equations (30) as well as

$$
\lambda_{1}^{\prime}=-\frac{\partial H}{\partial x_{1}}=\beta \sin \left(x_{1}\right), \quad \lambda_{2}^{\prime}=-\frac{\partial H}{\partial x_{2}}=-x_{2}-\lambda_{1} .
$$

The state and costate equations, (30) and (38), require four boundary conditions in order to obtain a solution to the optimal control problem. There are two boundary conditions given on the states, which must be supplemented by two transversality conditions on the costates.

For our elastic strut problem, $q=1$ and upon examining the Legendre-Clebsch condition we find that

$$
\begin{aligned}
& H_{u}=\lambda_{2}, \quad H_{u}^{\prime}=\lambda_{2}^{\prime}=-x_{2}-\lambda_{1}, \quad H_{u}^{\prime \prime}=-x_{2}^{\prime}-\lambda_{1}^{\prime}=-u-\beta \sin \left(x_{1}\right), \\
& \frac{\partial}{\partial u}\left(H_{u}^{\prime \prime}\right)=\frac{\partial}{\partial u}\left(-\beta \sin \left(x_{1}\right)-u\right)=-1 .
\end{aligned}
$$

\footnotetext{
${ }^{6}$ For further details on the role of the transversality conditions in prescribing boundary conditions, the reader is referred to $[1,11,20]$.

${ }^{7}$ For other examples featuring these conditions, the interested reader is referred to $[4,9]$.
} 
Hence, the condition is satisfied for all solutions, regardless of the boundary conditions used. Secondly, as $F_{\mathrm{xx}}=G_{\mathrm{xx}}=\left(v_{0} \phi_{\mathrm{x}}\right)_{\mathrm{x}}=\left(v_{f} \psi_{\mathrm{x}}\right)_{\mathrm{x}}=H_{u \mathrm{x}}=0$, the Jacobson condition is trivially satisfied. Finally, we can determine that the optimal control $u=u^{*}$ from (37) with the help of the intermediate results $(39)_{1,2,3}$ :

$$
u^{*}=-\beta \sin \left(x_{1}\right) \text {. }
$$

This prescription for the optimal control is valid regardless of the boundary conditions imposed on the strut.

In summary, the necessary conditions for an extremal $\left(\mathrm{x}^{*}, \lambda^{*}, u^{*}=-\beta \sin \left(x_{1}\right)\right)$ of $J$ are the satisfaction of

$$
\lambda_{1}^{\prime}=\beta \sin \left(x_{1}\right), \quad \lambda_{2}^{\prime}=-x_{2}-\lambda_{1}, \quad x_{1}^{\prime}=x_{2}, \quad x_{2}^{\prime}=-\beta \sin \left(x_{1}\right),
$$

subject to the boundary and transversality conditions

$$
\phi\left[x_{1}\left(s_{0}\right), x_{2}\left(s_{0}\right)\right]=0, \quad \psi\left[x_{1}\left(s_{f}\right), x_{2}\left(s_{f}\right)\right]=0,
$$

and

$$
\lambda\left(s_{0}\right)=\left.v_{0} \phi_{\mathrm{x}}\right|_{\mathrm{x}=\mathrm{x}^{*}\left(s_{0}\right)}, \quad \lambda\left(s_{f}\right)=\left.v_{f} \psi_{\mathrm{x}}\right|_{\mathrm{x}=\mathrm{x}^{*}\left(s_{f}\right)},
$$

respectively. Note that (42) and (43) constitute six equations. Two of these equations are used to determine $v_{0}$ and $v_{f}$ and the other four provide boundary conditions for (41).

\subsection{The Second Variation}

Prior to computing the second variation, it is convenient to define notations for the first derivatives of $\mathrm{f}$ and second derivatives of $L$ evaluated on the extremal:

$$
\begin{aligned}
& \mathrm{A}=\mathrm{f}_{\mathrm{x}}\left(\mathrm{x}^{*}, u^{*}\right), \quad \mathrm{B}=\mathrm{f}_{u}\left(\mathrm{x}^{*}, u^{*}\right), \\
& \mathrm{Q}=L_{\mathrm{xx}}\left(\mathrm{x}^{*}, u^{*}\right), \quad \mathrm{C}^{T}=L_{u \mathrm{x}}\left(\mathrm{x}^{*}, u^{*}\right), \quad \mathrm{R}=L_{u u}\left(\mathrm{x}^{*}, u^{*}\right)=0 .
\end{aligned}
$$

In addition, the derivatives of the initial and terminal costs and the transversality conditions will play a key role in the sequel:

$$
\begin{aligned}
& \mathrm{Q}_{0}=\left.\left(F+v_{0} \phi\right)_{\mathrm{xx}}\right|_{\left(\mathrm{x}=\mathrm{x}^{*}\left(s_{0}\right), u=u^{*}\left(s_{0}\right)\right)}, \\
& \mathrm{Q}_{f}=\left.\left(G+v_{f} \psi\right)_{\mathrm{xx}}\right|_{\left(\mathrm{x}=\mathrm{x}^{*}\left(s_{f}\right), u=u^{*}\left(s_{f}\right)\right)} .
\end{aligned}
$$

The second variation of the amended functional $\bar{J}$ can be expressed as

$$
\begin{aligned}
\delta^{2} \bar{J}= & \frac{1}{2} \int_{s_{0}}^{s_{f}} \delta \mathrm{x} \cdot(\mathrm{Q} \delta \mathrm{x})+2 \delta u \cdot(\mathrm{C} \delta \mathrm{x}) d s \\
& +\frac{1}{2} \delta \mathrm{x}\left(s_{0}\right) \cdot\left(\mathrm{Q}_{0} \delta \mathrm{x}\left(s_{0}\right)\right)+\frac{1}{2} \delta \mathrm{x}\left(s_{f}\right) \cdot\left(\mathrm{Q}_{f} \delta \mathrm{x}\left(s_{f}\right)\right),
\end{aligned}
$$

where the variations $\delta \mathbf{x}, \delta u$, and $\delta \lambda$ satisfy the differential equations and boundary conditions

$$
\begin{aligned}
& \delta \mathrm{x}^{\prime}=\mathrm{A} \delta \mathrm{x}+\mathrm{B} \delta u, \\
& \delta \lambda^{\prime}=-\mathrm{Q} \delta \mathrm{x}-\mathrm{C}^{T} \delta u+\mathrm{A}^{T} \delta \lambda, \\
& \left.\phi_{\mathrm{x}} \delta \mathrm{x}\left(s_{0}\right)\right|_{\left(\mathrm{x}=\mathrm{x}^{*}\left(s_{0}\right), u=u^{*}\left(s_{0}\right)\right)}=0, \\
& \left.\psi_{\mathrm{x}} \delta \mathrm{x}\left(s_{f}\right)\right|_{\left(\mathrm{x}=\mathrm{x}^{*}\left(s_{f}\right), u=u^{*}\left(s_{f}\right)\right)}=0 .
\end{aligned}
$$


As a final preliminary, we define the linearized Hamiltonian $h$ :

$$
h=\frac{1}{2} \delta \mathrm{x} \cdot(\mathrm{Q} \delta \mathrm{x})+\delta u \cdot(\mathrm{C} \delta \mathrm{x})+\delta \lambda \cdot(\mathrm{A} \delta \mathrm{x}+\mathrm{B} \delta u) .
$$

Following the classical treatment by Legendre [2], it is standard procedure to add an identity to $\delta^{2} \bar{J}$ :

$$
\begin{aligned}
0= & \frac{1}{2} \int_{s_{0}}^{s_{f}} \frac{d}{d s}(\delta \mathrm{x} \cdot(\mathrm{S}(s) \delta \mathrm{x})) d s+\frac{1}{2} \delta \mathrm{x}\left(s_{0}\right) \cdot\left(\mathrm{S}\left(s_{0}\right) \delta \mathrm{x}\left(s_{0}\right)\right) \\
& -\frac{1}{2} \delta \mathrm{x}\left(s_{f}\right) \cdot\left(\mathrm{S}\left(s_{f}\right) \delta \mathrm{x}\left(s_{f}\right)\right) .
\end{aligned}
$$

The matrix $\mathrm{S}=\mathrm{S}^{T}$ is assumed to be a differentiable function of $s$. Evaluating $\delta \mathbf{x}^{\prime}$ using $(48)_{1,2}$, and combining terms, we find that $\delta^{2} \bar{J}$ transforms to

$$
\begin{aligned}
\delta^{2} \bar{J}= & \frac{1}{2} \int_{s_{0}}^{s_{f}} \delta \mathrm{x} \cdot\left(\left(\mathrm{S}^{\prime}+\mathrm{Q}+\mathrm{SA}+\mathrm{A}^{T} \mathrm{~S}\right) \delta \mathrm{x}\right)+2 \delta u \cdot\left(\left(\mathrm{C}+\mathrm{B}^{T} \mathrm{~S}\right) \delta \mathrm{x}\right) d s \\
& +\frac{1}{2} \delta \mathrm{x}\left(s_{0}\right) \cdot\left(\left(\mathrm{Q}_{0}+\mathrm{S}\left(s_{0}\right)\right) \delta \mathrm{x}\left(s_{0}\right)\right) \\
& +\frac{1}{2} \delta \mathrm{x}\left(s_{f}\right) \cdot\left(\left(\mathrm{Q}_{f}-\mathrm{S}\left(s_{f}\right)\right) \delta \mathrm{x}\left(s_{f}\right)\right) .
\end{aligned}
$$

It follows that a sufficient condition for positive semi-definiteness of $\delta^{2} \bar{J}$ is ${ }^{8}$

$$
\begin{aligned}
\mathrm{S}^{\prime}+\mathrm{Q}+\mathrm{SA}+\mathrm{A}^{T} \mathrm{~S} & \geq 0, \\
\mathrm{C}+\mathrm{B}^{T} \mathrm{~S} & =0, \\
\delta \times\left(s_{0}\right) \cdot\left(\left(\mathrm{Q}_{0}+\mathrm{S}\left(s_{0}\right)\right) \delta \times\left(s_{0}\right)\right)+\delta \times\left(s_{f}\right) \cdot\left(\left(\mathrm{Q}_{f}-\mathrm{S}\left(s_{f}\right)\right) \delta \times\left(s_{f}\right)\right) & \geq 0 .
\end{aligned}
$$

We note that the variations $\delta \mathbf{x}\left(s_{0, f}\right)$ in $(52)_{3}$ also need to satisfy the linearized transversality conditions $(48)_{3,4}$. This significantly reduces the number of restrictions on the matrices $Q_{0}+$ $\mathrm{S}\left(s_{0}\right)$ and $\mathrm{Q}_{f}-\mathrm{S}\left(s_{f}\right)$.

It has been shown by Bell and Jacobson [1] that a matrix $S$ which satisfies (52) 1 solves the Riccati differential equation ${ }^{9}$

$$
\mathrm{S}^{\prime}+\mathrm{Q}+\mathrm{SA}+\mathrm{A}^{T} \mathrm{~S}+\mathrm{M}^{T}\left(\frac{\partial h_{u}^{\prime \prime}}{\partial u}\right)^{-1} \mathrm{M}=0,
$$

where

$$
\begin{aligned}
\frac{\partial h_{u}^{\prime \prime}}{\partial \delta u} & =\frac{\partial}{\partial \delta u}\left(\frac{\partial^{3} h}{\partial s \partial s \partial \delta u}\right) \\
& =\mathrm{CAB}+\mathrm{B}^{T} \mathrm{~A}^{T} \mathrm{C}^{T}+\mathrm{C}^{\prime} \mathrm{B}-\left(\mathrm{B}^{\prime}\right)^{T} \mathrm{C}^{T}-\mathrm{B}^{T} \mathrm{QB},
\end{aligned}
$$

\footnotetext{
${ }^{8}$ We use the standard notation to show that a matrix $P$ is positive semi-definite by the expression $P \geq 0$.

${ }^{9}$ An alternative prescription for a Riccati equation of this type was presented in McDanell and Powers [18]. For the elastica problem of interest in the present paper, the Riccati equations proposed in [1] and [18] are identical.
} 


$$
\mathrm{M}=\left(\mathrm{AB}-\mathrm{B}^{\prime}\right)^{T} \mathrm{~S}+\mathrm{B}^{T} \mathrm{Q}-\mathrm{CA}-\mathrm{C}^{\prime} .
$$

The boundary conditions on $S$ in the Riccati differential equation (53) are obtained by examining $(52)_{2,3}$. To this end, we first note that Bell and Jacobson [1] also showed that for $(52)_{2}$ to be satisfied, it is necessary and sufficient that

$$
\mathrm{C}\left(s_{f}\right)+\mathrm{B}^{T}\left(s_{f}\right) \mathrm{S}\left(s_{f}\right)=0 .
$$

We will use (52) $)_{3}$ and (55) to determine the boundary conditions for solutions to (53).

\subsubsection{Application to the Elastica}

For the problem of interest, many of the matrices featuring in the second variation are simple:

$$
\mathrm{A}=\left[\begin{array}{ll}
0 & 1 \\
0 & 0
\end{array}\right], \quad \mathrm{B}=\left[\begin{array}{l}
0 \\
1
\end{array}\right], \quad \mathrm{C}=0, \quad \mathrm{Q}=\left[\begin{array}{cc}
-\beta \cos \left(x_{1}\right) & 0 \\
0 & 1
\end{array}\right] .
$$

In addition,

$$
\frac{\partial h_{u}^{\prime \prime}}{\partial \delta u}=-\mathrm{B}^{T} \mathrm{QB}=-1, \quad \mathrm{M}=\left[\begin{array}{ll}
S_{11} & S_{12}+1
\end{array}\right] .
$$

For all the problems of interest, (55) implies that

$$
S_{12}\left(s_{f}\right)=0, \quad S_{22}\left(s_{f}\right)=0 .
$$

The remaining boundary value is obtained from the condition $(52)_{3}$. These conditions depend on the boundary conditions for the elastic strut, and we defer imposing them until later.

With the help of (56) and (57), the Riccati differential equations can be computed from (53):

$$
S_{11}^{\prime}=S_{11}^{2}+\beta \cos \left(x_{1}\right), \quad S_{12}^{\prime}=S_{11} S_{12}, \quad S_{22}^{\prime}=S_{12}^{2} .
$$

Subject to (58), these equations have the solution

$$
S_{11}(s)=r(s, c, \beta), \quad S_{12}(s)=0, \quad S_{22}(s)=0,
$$

where $c$ is a constant. Remarkably then, the issue of the minimization of the second variation boils down to a single Riccati equation (59) $)_{1}$, which is identical to (17) that we found earlier in Sect. 3 using a different variational principle. Thus, if we can find a bounded solution $S_{11}(s)$ to $(59)_{1}$, then we will have established sufficient conditions for the energy $I$ (which is equivalent to $E$ ) to be minimized and necessary conditions for $J$ to be minimized.

In the special case where $\cos \left(x_{1}\right)=0$, the function $r(s, c, \beta)$ can be easily computed:

$$
r(\tau, c, \beta)=\sqrt{\beta} \tan (\sqrt{\beta}(\tau-c)) .
$$

This solution is shown in Fig. 2 for two cases. Of particular importance in the results shown in this figure are the unboundedness of $r$ when $\beta$ has certain critical values:

$$
\lim _{x \rightarrow \pm 0.5} r\left(x, 0.5, \beta=\pi^{2}\right)= \pm \infty, \quad \lim _{x \rightarrow 0} r\left(x, 1.0, \beta=\frac{\pi^{2}}{4}\right)=-\infty .
$$

The solutions shown in Fig. 2(a) feature in the stability analysis of the straight fixed-fixed strut, while those shown in Fig. 2(b) feature in the stability analysis of the straight fixed-free strut. 

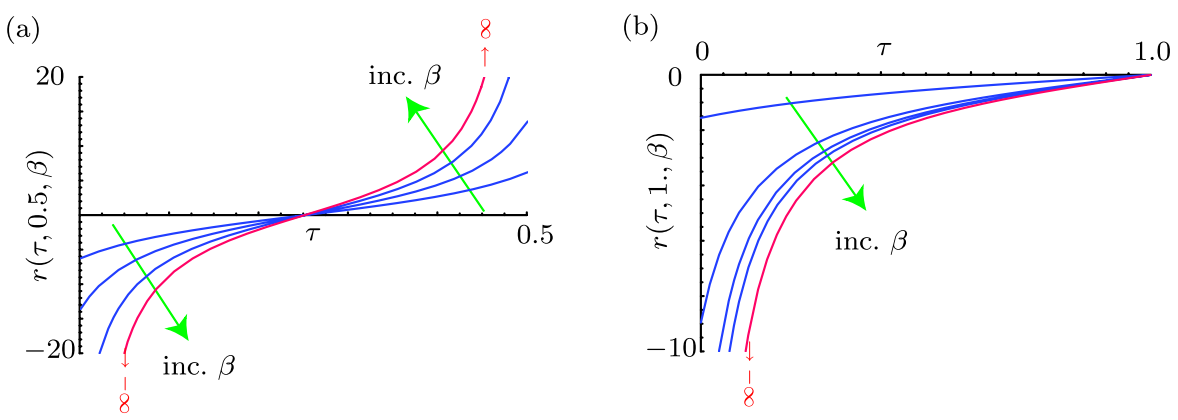

Fig. 2 Solutions (61) to the Riccati equation (60) when $\cos \left(x_{1}\right)=1.0$ for (a) the case where $c=0.5$ and $\tau \in[-0.5,0.5]$, and (b) $c=1.0$ and $\tau \in[0,1.0]$. In (a), $\beta=4,6,8$, and $\pi^{2}$. In (b), $\beta=1,2,2.2,2.3$, and $\frac{\pi^{2}}{4}$

\section{Three Simple Buckling Problems}

We now explore the application of the necessary and sufficient conditions to the buckling problems. Recall that the classical formulation discussed in Sect. 3.1 leads to identical extrema as the control-based formulation. Furthermore, the necessary condition J1 featuring the search for a solution to (17) subject to (18) results in the same conclusions as the investigation of the Riccati equation (60) derived in Sect. 4.2.1. Consequently, we restrict our discussion to examining the solutions of (41) and the implications of finding solutions to (60).

\subsection{The Fixed-Fixed Strut}

For the case where Dirichlet boundary conditions are applied at both ends of the rod, the boundary conditions for (41) are determined from the boundary conditions

$$
\phi=x_{1}(0), \quad \psi=x_{1}(1),
$$

with the help of (42) and (43). It is easy to evaluate (43) to find that

$$
\left[\lambda_{1}(0) \quad \lambda_{2}(0)\right]=v_{0}\left[\begin{array}{ll}
1 & 0
\end{array}\right], \quad\left[\lambda_{1}(1) \quad \lambda_{2}(1)\right]=v_{f}\left[\begin{array}{ll}
1 & 0
\end{array}\right]
$$

Thus, we find the four boundary conditions

$$
\begin{array}{ll}
x_{1}(0)=0, & x_{1}(1)=0, \\
\lambda_{2}(0)=0, & \lambda_{2}(1)=0 .
\end{array}
$$

The remaining two conditions from $(64), \lambda_{1}(0)=v_{0}$ and $\lambda_{1}(1)=v_{f}$, are used to determine $v_{0}$ and $v_{f}$ from the solutions to (41).

Fixing the value of $\beta$ to be greater than the known critical buckling value $\left(\beta=\pi^{2}\right)$, we obtain solutions to (41) subject to (65). These are graphically represented in Fig. 3, and a bifurcation diagram showing the behavior of all possible equilibria is presented in Fig. 4. In the bifurcation diagram, the dimensionless energy $\bar{E}=I$ (cf. (10)). The displacement $y$ 

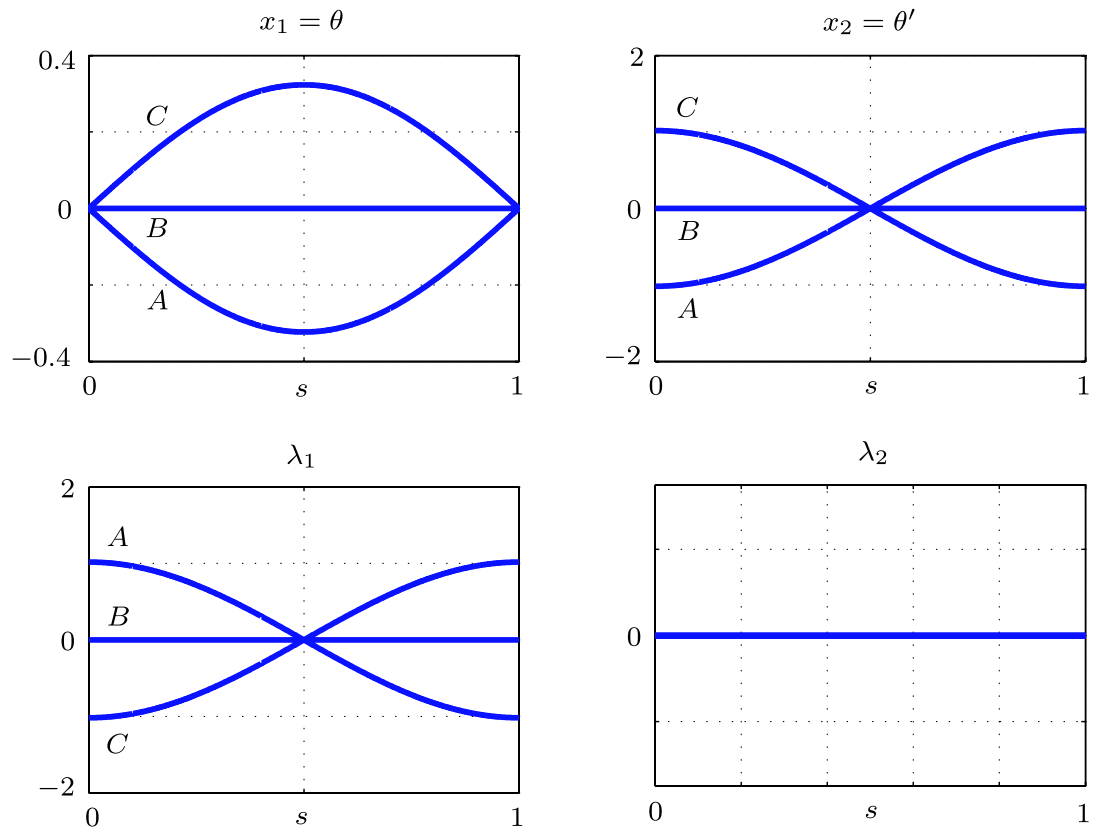

Fig. 3 Solutions $x_{1}(s)=\theta(s), x_{2}(s)=\theta^{\prime}(s), \lambda_{1}(s)$, and $\lambda_{2}(s)$ to the state and costate equations (41) with $\beta=10$ and Dirichlet-Dirichlet boundary conditions (65). Solutions $A, B$, and $C$ correspond to the points in Fig. 4

Fig. 4 Bifurcation diagram showing the behavior of all equilibrium solutions for the Dirichlet-Dirichlet case over the region $\beta=[0,60]$

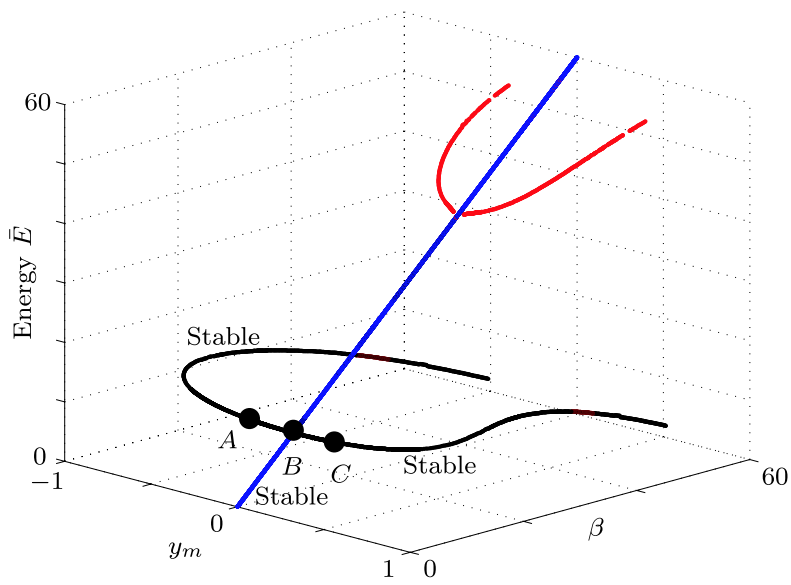

is the signed maximum lateral distance that the rod is displaced when it deforms to either postbuckled state and is defined by

$$
y_{m}=\operatorname{sgn}(Y)(\max |Y / \ell|),
$$

where $Y$ is the Cartesian coordinate defined by $(Y / \ell)^{\prime}=\sin (\theta)$. 
(a)

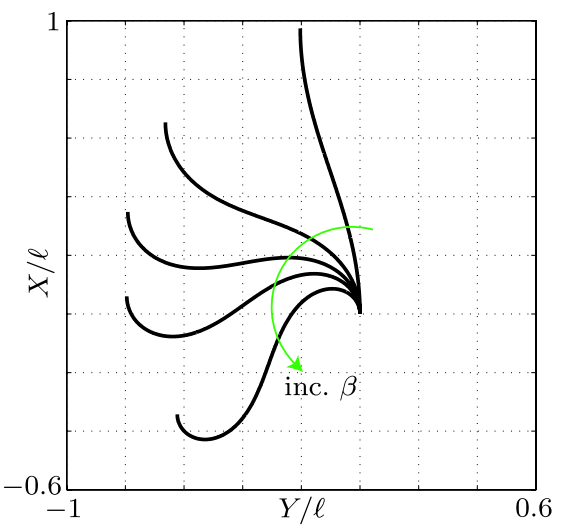

(b)

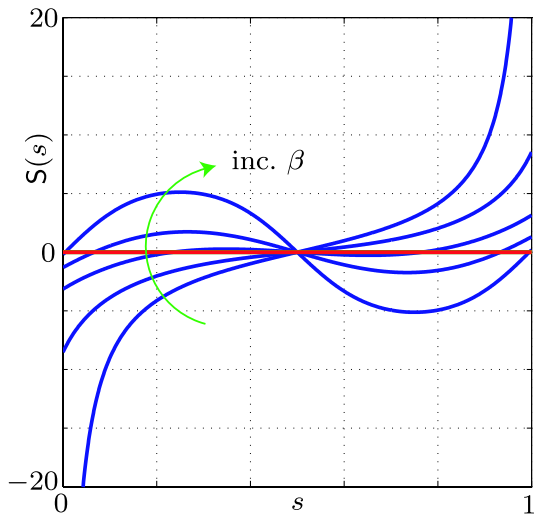

Fig. 5 (a) Buckled solutions to the state equations, which reside on branch A-C in Fig. 4; (b) Solutions to the Riccati equation (59) for each buckled configuration. In (b), $S_{12}(s)=S_{22}(s)=0$ in all cases. All other solutions are $S_{11}(s)$. In both (a) and (b), $\beta=10,12,15,20$, and 40

For the Dirichlet-Dirichlet case, the boundary conditions on the variations are determined with the help of $(48)_{3,4}$ :

$$
\delta x_{1}\left(s_{0}\right)=0, \quad \delta x_{1}\left(s_{f}\right)=0 .
$$

Consequently, the conditions $(52)_{3,4}$ simplify dramatically to

$$
S_{22}\left(s_{0}\right)\left(\delta x_{2}\left(s_{0}\right)\right)^{2} \geq 0, \quad-S_{22}\left(s_{f}\right)\left(\delta x_{2}\left(s_{f}\right)\right)^{2} \geq 0 .
$$

With the help of the solution $(60)_{2,3}$ to the Riccati equation, we find that these two conditions are identically satisfied. It remains to find a solution to the Riccati equation (59) where $S_{11}(s)$ is finite. Restricting attention to the straight strut, we find that a solution

$$
S_{11}(s)=\sqrt{\beta} \tan (\sqrt{\beta}(s-0.5))
$$

exists provided $\beta<\pi^{2}$ (cf. Fig. 2(a) and (61)). When $\beta \geq \pi^{2}$, the bounded solution for all $s \in[0,1]$ does not exist. Consequently, the sufficient condition recovers the classical result that a strut with fixed-fixed boundary conditions is stable provided $\beta<\pi^{2}$. These results agree with those obtained using the condition J1 by Born [3], among others (see, e.g., [16]).

For the buckled configurations (i.e., solutions along the branch containing points A and $\mathrm{C}$ in Fig. 4), the Riccati equation (59) must be solved numerically. A variety of buckled configurations, along with their respective Riccati solutions, are graphically represented in Fig. 5. The key feature in Fig. 5(b) is that each solution $S_{11}(s)$ is finite, implying that every buckled solution featured in Fig. 5(a) is stable. For the straight strut and all subsequent branches beyond point B in Fig. 4, no solutions to the Riccati equation could be computed, which strongly suggests instability of these rod configurations.

\subsection{The Fixed-Free Strut}

For the case where there is a Dirichlet boundary condition at the beginning of the rod and a Neumann boundary condition at the end (i.e., fixed-free), we have

$$
\phi=x_{1}(0), \quad \psi=x_{2}(1) .
$$



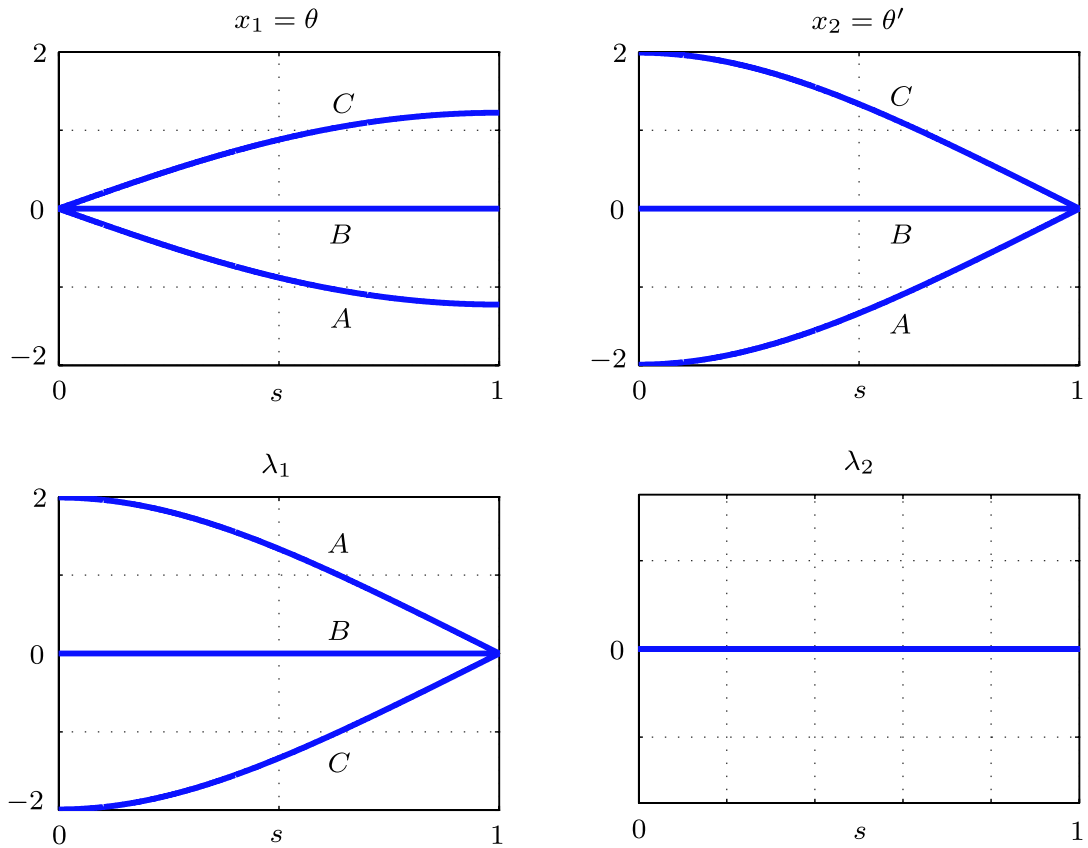

Fig. 6 Solutions $x_{1}(s)=\theta(s), x_{2}(s)=\theta^{\prime}(s), \lambda_{1}(s)$, and $\lambda_{2}(s)$ to the state and costate equations (41) with $\beta=2.5$ and Dirichlet-Neumann boundary conditions (71). Solutions $A, B$, and $C$ correspond to the points in Fig. 7

As with the previous case, we invoke the help of (42) and (43) to find four boundary conditions:

$$
\begin{array}{ll}
x_{1}(0)=0, & x_{2}(1)=0, \\
\lambda_{1}(1)=0, & \lambda_{2}(0)=0 .
\end{array}
$$

These four conditions are supplemented by the conditions $\lambda_{1}(0)=v_{0}$ and $\lambda_{2}(1)=v_{f}$. As before, $v_{0}$ and $v_{f}$ are determined from the solutions to (41). The solutions of equations (41) subject to (71) are shown in Fig. 6 and its bifurcation diagram is shown in Fig. 7.

For the Dirichlet-Neumann case, the boundary conditions on the variations are

$$
\delta x_{1}\left(s_{0}\right)=0, \quad \delta x_{2}\left(s_{f}\right)=0 .
$$

Consequently, the conditions $(52)_{3,4}$ simplify to

$$
S_{22}\left(s_{0}\right)\left(\delta x_{2}\left(s_{0}\right)\right)^{2} \geq 0, \quad-S_{11}\left(s_{f}\right)\left(\delta x_{1}\left(s_{f}\right)\right)^{2} \geq 0 .
$$

With the help of the solution (60) to the Riccati equation, we find that these two conditions are satisfied if we can find a solution to the Riccati equation where $S_{11}\left(s_{f}\right) \leq 0$ and $S_{11}(s)$ is finite. Restricting attention to the straight strut, we find that a solution

$$
S_{11}(s)=\sqrt{\beta} \tan (\sqrt{\beta}(s-1))
$$

exists provided $\beta<\frac{\pi^{2}}{4}$ (cf. Fig. 2(b) and (61)). When $\beta \geq \frac{\pi^{2}}{4}$, the bounded solution for all $s \in[0,1]$ does not exist. Consequently, the L1 condition recovers the classical result that a 
Fig. 7 Bifurcation diagram showing solution behavior for the Dirichlet-Neumann case over the region $\beta=[0,60]$

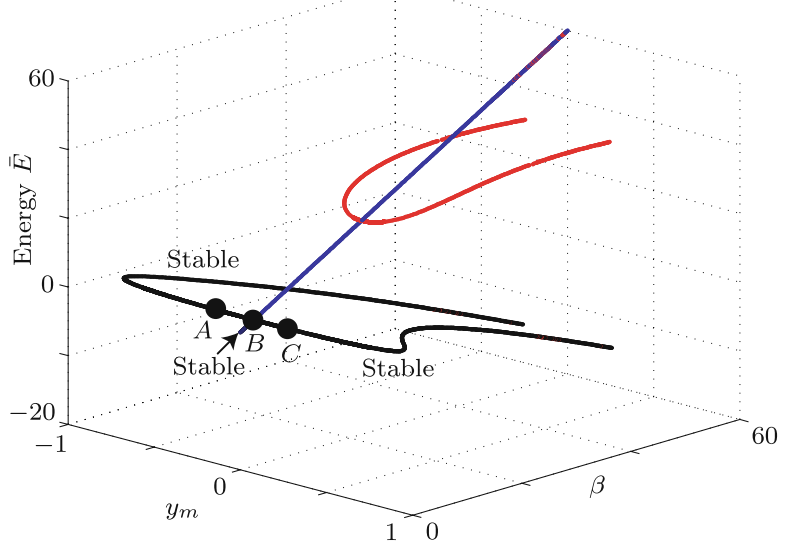

(b)

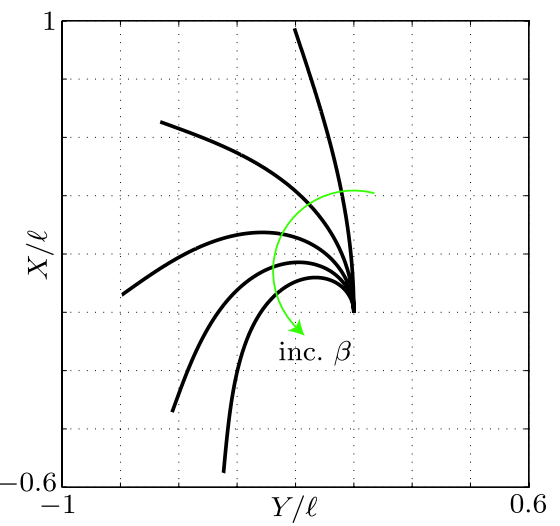

Fig. 8 (a) Buckled solutions to the state equations, which reside on branches A \& C in Fig. 7; (b) Solutions to the Riccati equation (59) for each buckled configuration. In (b), $S_{12}(s)=S_{22}(s)=0$ in all cases. All other solutions are $S_{11}(s)$. In both (a) and (b), $\beta=2.5,3,5,10$, and 20

strut with fixed-free boundary conditions is stable provided $\beta<\frac{\pi^{2}}{4}$. This result can be found Born [3, Sect. 9], who used the condition J1 to establish the bound on $\beta$.

Just as in the fixed-fixed case, solutions to the Riccati equation (59) are graphically represented in Fig. 8 for the branch of solutions labeled A and C in Fig. 7. As before, the solutions in Fig. 8(b) are finite, meaning the configurations in Fig. 8(a) are stable for the fixed-free case. Similarly, no Riccati solutions could be computed for the straight strut and subsequent branches beyond point B in Fig. 7, which strongly suggests instability for these rod configurations.

\subsection{The Free-Free Strut}

The final case of interest arises when both boundary conditions are Neumann:

$$
\phi=x_{2}(0), \quad \psi=x_{2}(1) .
$$


(a)
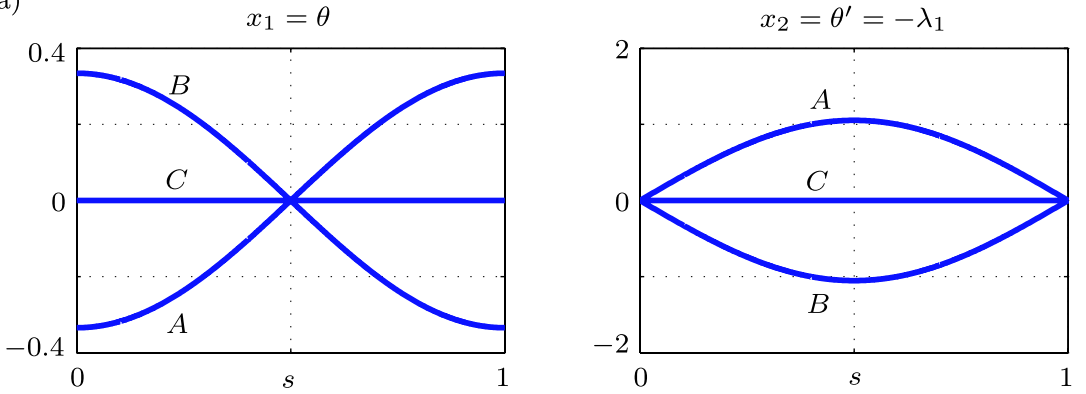

(b)

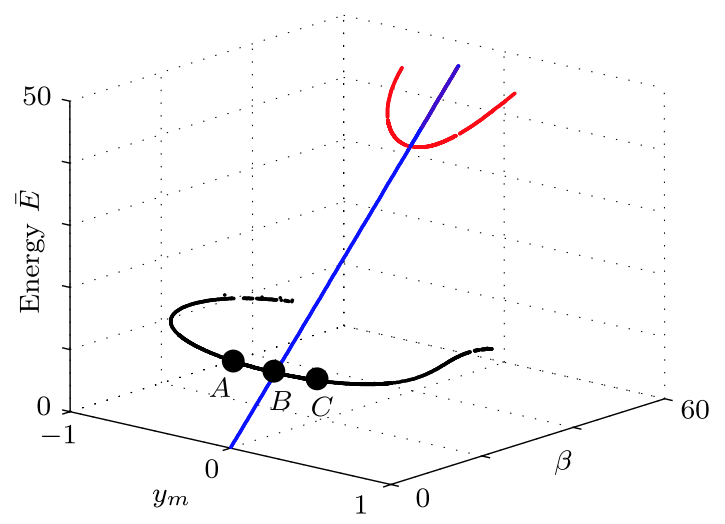

Fig. 9 (a) Solutions $x_{1}(s)=\theta(s), x_{2}(s)=\theta^{\prime}(s)$, and $\lambda_{1}(s)$ to the state and costate equations (41) with $\beta=10$ and Neumann-Neumann boundary conditions (76). The solution to the second costate equation is satisfied by $\lambda_{2}=\Lambda$, where $\Lambda$ is an arbitrary constant. (b) Bifurcation diagram showing solution behavior for the Neumann-Neumann case over the region $\beta=[0,60]$

As with the two previous cases, we invoke the help of (42) and (43) to find the four boundary conditions:

$$
\begin{array}{ll}
x_{2}(0)=0, & x_{2}(1)=0, \\
\lambda_{1}(0)=0, & \lambda_{1}(1)=0 .
\end{array}
$$

We also find the conditions $\lambda_{2}(0)=v_{0}$ and $\lambda_{2}(1)=v_{f}$, where $v_{0}$ and $v_{f}$ are to be solved in the usual manner.

Equations (41) and (76) are not well-posed. However, we can first solve for $x_{1}, x_{2}$, and $\lambda_{1}$. To do so, we require only one of the transversality conditions $(76)_{3,4}$, and so we arbitrarily select (76) 3 . The resulting solutions and bifurcation diagram are shown in Fig. 9. It is worth noting that the transversality condition that was eliminated is still satisfied in the solution $\left(\lambda_{1}(1)=0\right)$. Furthermore,

$$
\lambda_{2}(s)=\Lambda
$$

where $\Lambda$ is an arbitrary constant. Thus $v_{f}=v_{0}$ are also arbitrary.

For the free-free strut, the boundary conditions on the variations are

$$
\delta x_{2}\left(s_{0}\right)=0, \quad \delta x_{2}\left(s_{f}\right)=0 .
$$


As in the previous two cases, the conditions $(52)_{3,4}$ simplify considerably:

$$
S_{11}\left(s_{0}\right)\left(\delta x_{1}\left(s_{0}\right)\right)^{2} \geq 0, \quad-S_{11}\left(s_{f}\right)\left(\delta x_{1}\left(s_{f}\right)\right)^{2} \geq 0 .
$$

With the help of the solution (60) to the Riccati equation (59), we find that we require $r(s, c, \beta)=0$. However, even for the trivial case where the rod is straight and $\cos \left(x_{1}\right)=1$, $S_{11}(s)=0$ does not satisfy (59). Consequently, the necessary condition J1 cannot be applied to the free-free beam. As remarked earlier in Sect. 3.2, application of the Jacobi condition $\mathrm{J} 1$ to the free-free strut is not valid [16]. However, the Riccati equation illustrates this in a transparent manner.

\section{Closing Remarks}

Our treatment of the fixed-fixed strut features a Riccati equation,

$$
w^{\prime}=w^{2}+\beta \cos \left(\theta^{*}\right)
$$

whereas traditional approaches such as $[8,16]$ have solved the Jacobi equation,

$$
\eta^{\prime \prime}+\beta \cos \left(\theta^{*}\right) \eta=0
$$

and examined conjugate points to $s=s_{0}=0$ on the interval $s \in(0,1]$. We have shown that the stability results that can be inferred from the L1 and J1 conditions are equivalent. In addition, for the free-free strut, the lack of a solution to the Riccati equation is a clear indication of the difficulties associated with this case. In contrast, the inapplicability of the $\mathrm{J} 1$ condition to the free-free strut is subtle and could easily be overlooked.

For the fixed-free case, L1 is much easier to use. In this case, the boundary condition of $w$ is simply on $w(s=1)=0$, which makes integrating the Riccati equation trivial. It is this feature of the Legendre condition that we are currently exploiting in examining the stability of static equilibria of branched elastic rods. In a work in progress, we find it necessary to solve a series of coupled Riccati equations (one for each branch) in order to establish stability of the branched structure.

Acknowledgements This research was partially supported by grant number CMMI-0726675 from the U.S. National Science Foundation. The authors are also grateful for several helpful comments from Prof. George Leitmann, Prof. Robert S. Manning, Prof. John Prussing, Prof. Florian Wagener, and Prof. Vera M. Zeidan.

Open Access This article is distributed under the terms of the Creative Commons Attribution Noncommercial License which permits any noncommercial use, distribution, and reproduction in any medium, provided the original author(s) and source are credited.

\section{References}

1. Bell, D.J., Jacobson, D.H.: Singular Optimal Control Problems. Academic Press, London (1975)

2. Bolza, O.: Lectures on the Calculus of Variations. University of Chicago Press, Chicago (1907)

3. Born, M.: Untersuchungen über die Stabilität der elastischen Linie in Ebene und Raum, unter verschiedenen Grenzbedingungen. Dieterichsche Universitäts-Buchdruckerei, Göttingen (1906)

4. Bryson, A.E. Jr., Ho, Y.C.: Applied Optimal Control: Optimization, Estimation, and Control. Hemisphere Publishing Corporation, Washington (1975). Revised printing 
5. Faruk Senan, N.A., O'Reilly, O.M., Tresierras, T.N.: Modeling the growth and branching of plants: a simple rod-based model. J. Mech. Phys. Solids 56(10), 3021-3036 (2008). doi:10.1016/ j.jmps.2008.06.005

6. Gelfand, I.M., Fomin, S.V.: Calculus of Variations. Prentice Hall, Englewood Cliffs (1964)

7. Jacobson, D.H.: A new necessary condition of optimality for singular control problems. SIAM J. Control 7, 578-595 (1969). doi:10.1137/0307042

8. Jin, M., Bao, Z.B.: Sufficient conditions for stability of Euler elasticas. Mech. Res. Commun. 35(3), 193-200 (2008). doi:10.1016/j.mechrescom.2007.09.001

9. Johnson, C.D., Gibson, J.E.: Singular solutions in problems of optimal control. IEEE Trans. Autom. Control AC-8, 4-15 (1963)

10. Kuznetsov, V.V., Levyakov, S.V.: Complete solution of the stability problem for elastica of Euler's column. Int. J. Non-Linear Mech. 37(6), 1003-1009 (2002). doi:10.1016/S0020-7462(00)00114-1

11. Leitmann, G.: The Calculus of Variations and Optimal Control. Plenum, New York (1981)

12. Levyakov, S.V.: Stability analysis of curvilinear configurations of an inextensible elastic rod with clamped ends. Mech. Res. Commun. 36(5), 612-617 (2009). doi:10.1016/j.mechrescom.2009.01.005

13. Levyakov, S.V., Kuznetsov, V.V.: Stability analysis of planar equilibrium configurations of elastic rods subjected to end loads. Acta Mech. 211(1-2), 73-87 (2010). doi:10.1007/s00707-009-0213-0

14. Love, A.E.H.: A Treatise on the Mathematical Theory of Elasticity, 4th edn. Cambridge University Press, Cambridge (1927)

15. Maddocks, J.H.: Stability of nonlinearly elastic rods. Arch. Ration. Mech. Anal. 85(4), 311-354 (1984). doi:10.1007/BF00275737

16. Manning, R.S.: Conjugate points revisited and Neumann-Neumann problems. SIAM Rev. 51(1), 193212 (2009). doi:10.1137/060668547

17. Manning, R.S., Rogers, K.A., Maddocks, J.H.: Isoperimetric conjugate points with application to the stability of DNA minicircles. Proc. R. Soc. Lond., Ser. A, Math. Phys. Eng. Sci. 454(1980), 3047-3074 (1998). doi:10.1098/rspa.1998.0291

18. McDanell, J.P., Powers, W.F.: New Jacobi-type necessary and sufficient conditions for singular optimization problems. AIAA J. 8, 1416-1420 (1970). doi:10.2514/3.5917

19. O'Reilly, O.M., Tresierras, T.N.: On the static equilibria of branched elastic rods. Int. J. Eng. Sci. 49(2), 212-227 (2011). doi:10.1016/j.ijengsci.2010.11.008

20. Pedregal, P.: Introduction to Optimization. Springer, New York (2004)

21. Rosenblueth, J.F.: Conjugate journey in optimal control. Int. Math. Forum 2(13-16), 633-674 (2007). http://www.m-hikari.com/imf-password2007/13-16-2007/index.html

22. Sachkov, Y.L.: Optimality of Euler elasticae. Dokl. Akad. Nauk 417(1), 23-25 (2007). doi:10.1134/ S106456240706004X

23. Sachkov, Y.L.: Conjugate points in the Euler elastic problem. J. Dyn. Control Syst. 14(3), 409-439 (2008). doi:10.1007/s10883-008-9044-X

24. Zeidan, V.: Sufficient conditions for variational problems with variable endpoints: Coupled points. Appl. Math. Optim. 27(2), 191-209 (1993). doi:10.1007/BF01195982 